\title{
Anisotropic Step-Flow Growth and Island Growth of GaN(0001) by Molecular Beam Epitaxy
}

\author{
M. H. Xie, S. M. Seutter, W. K. Zhu, L. X. Zheng, Huasheng Wu, and S. Y. Tong \\ Department of Physics, The University of Hong Kong, Pokfulam Road, Hong Kong
}

(Received 30 October 1998)

\begin{abstract}
Anisotropic growth is observed for $\mathrm{GaN}(0001)$ during molecular beam epitaxy for both the stepflow growth mode and two-dimensional (2D) nucleation growth mode. Using scanning tunneling microscopy, we find that in the step-flow growth mode, growth anisotropy strongly influences the shape of terrace edges, making them strikingly different between hexagonal and cubic films. In the 2D nucleation growth mode, anisotropic growth results in triangularly shaped islands. The importance of understanding growth anisotropy to achieve better grown GaN films is discussed. [S0031-9007(99)08731-1]
\end{abstract}

PACS numbers: 68.55.Jk, 61.16.Ch, 68.35.Bs, 68.35.Fx

The growth of GaN thin films has attracted intense recent interest, because important high-performance GaNbased devices have been fabricated [1-3]. For device fabrication, the thin films are grown mostly by metalorganic vapor phase epitaxy (MOVPE). On the other hand, the use of molecular beam epitaxy (MBE) to study GaN growth has become increasingly popular because of the ease to incorporate in situ ultrahigh vacuum (UHV) characterization tools $[4,5]$. Up to now, there has been little known concerning the microscopic aspects of growth mechanism of GaN films.

We report here the observation of anisotropic growth behavior of $\mathrm{GaN}(0001)$ thin films using radio frequency (rf) plasma assisted MBE. By changing the growth temperature, we observe anisotropic growth behavior in both the step-flow growth mode and 2D island growth mode. The speed in which the edge of step or island grows is mainly controlled by two factors: (i) the ease in which an arriving species (adatom) binds to a site at the edge [6] and (ii) the subsurface influenced diffusion rate of an adatom along an edge [7]. The former is mainly a local bonding interaction between an adatom and edge atoms. The latter is the influence of subsurface atoms on the mobility of an adatom. We shall use a microscopic picture to explain the experimentally observed growth anisotropy and indicate which of the above two processes is dominant in the $\mathrm{GaN}(0001)$ case.

The MBE system consists of a deposition chamber equipped with reflection high-energy electron diffraction (RHEED) and connected under UHV conditions to three other chambers equipped with various characterization tools. These include a variable-temperature scanning tunneling microscopy (STM), atomic force microscopy (AFM), low-energy electron diffraction (LEED), and x-ray photoemission spectroscopy (XPS). A Ga flux is supplied by a conventional Knudsen cell, and a $\mathrm{N}$ flux is provided by an Oxford plasma unit. Typical pressure in the MBE chamber during deposition is $5 \times 10^{-5}$ torr at a $\mathrm{N}_{2}$ flow rate of 0.13 standard cubic centimeters per minute. The $6 \mathrm{H}$-SiC $(0001)$ substrate (Cree) is diced into $11 \times 3 \mathrm{~mm}^{2}$ rectangular pieces. It is degreased in acetone and alcohol before being loaded into the vacuum. The surface is prepared in the UHV at $1100{ }^{\circ} \mathrm{C}$ under a flux of $\mathrm{Si}$. This procedure results in a $(\sqrt{3} \times$ $\sqrt{3}) R 30^{\circ}$ reconstruction on its Si face. STM pictures show atomically flat terraces of more than $1000 \AA$ wide [8]. On top of such a surface, a thick $(\sim 1 \mu \mathrm{m}) \mathrm{GaN}$ buffer layer is grown at $650{ }^{\circ} \mathrm{C}$. A Ga/N flux ratio of approximately 2 is maintained during growth, where the effective fluxes of $\mathrm{Ga}$ and $\mathrm{N}$ are calibrated by growth rates of $\mathrm{GaN}$ in the Ga-limited and N-limited regimes, respectively. The growth rates are measured by RHEED intensity oscillations and cross checked by total thickness measurements. The typical growth rate is $0.26 \AA / \mathrm{sec}$. The atomic order of the substrate surface on which the growth starts and the $\mathrm{Ga} / \mathrm{N}$ flux ratio are important parameters to achieve a flat surface morphology. Previous studies using different growth conditions have reported 3D columnar growth [9].

Further growth on such a thick GaN buffer is studied both at low temperature $\left(500{ }^{\circ} \mathrm{C}\right)$ and high temperature $\left(650{ }^{\circ} \mathrm{C}\right)$. Low temperature growth is shown to result in 2D islands, while high temperature leads to the step-flow growth mode. To observe surface morphology by STM, the growing surface is thermally quenched at the same time when the growth is stopped. To stop the growth, the $\mathrm{Ga}$ and $\mathrm{N}$ shutters are closed simultaneously together with the switching off of the plasma generator's power supply. Sample thermal quenching is achieved by switching off the dc power to the substrate. Since sample heating is by direct current, the sample cools down rapidly once the current is stopped. From RHEED, the surface of the film shows a " $1 \times 1$ " pattern. The film is determined to have a Ga polarity [i.e., (0001) face], because we have varied the Ga coverage on such samples and reproduced the reconstruction structures reported by Smith et al. on this face [10].

In the following, we shall describe first the surface morphology of the GaN film under step-flow growth. Generally, steps act as sinks of adatoms. When the diffusion length of an adatom is long compared to the terrace width, a step-flow growth mode is normally 
achieved where the growth is seen by the advance of steps and no island is nucleated. An STM image of a GaN surface grown in the step-flow growth mode at $650{ }^{\circ} \mathrm{C}$ is shown in Fig. 1. A number of terraces, some as wide at $1000 \AA$, are clearly visible. A most striking feature in this picture is that as one descends the terraces along a symmetry crystallographic direction, e.g., $\left[\begin{array}{llll}1 & 0 & 1 & 0\end{array}\right]$, the step edges alternate between jagged and smooth. On a given terrace, a smooth edge becomes a jagged edge by changing a $60^{\circ}$ direction.

To explain this rather unusual step-edge anisotropy, we refer to the atomic configuration of a (111) bilayer lattice shown in Fig. 2(a). We label the symmetry crystallographic directions in both the fcc(111) (diamond or zinc blende) and hcp(0001) (wurtzite) conventions. Considering only the dangling bonds in the bilayer, we see that there are two types of step edges: Type $A$ edge, normal to [ $\overline{1} \overline{1} 2]$ or equivalent directions, is characterized by two dangling bonds per edge atom and type $B$ edge, normal to [ $\left.\begin{array}{lll}2 & 1 & 1\end{array}\right]$ or equivalent directions, is characterized by one dangling bond per edge atom. It is reasonable to assume, and we shall show later that this assumption is correct, that a type $A$ edge will grow faster than a type $B$ edge because of the greater tendency for an arriving adatom to bind to a type $A$ edge. If the bilayer is an island on a surface, the faster growth speed in the direction normal to a type $A$ edge will ultimately result in a triangularly shaped island whose vertices point along the [ $\left.\begin{array}{lll}1 & 1 & 2\end{array}\right]$ and equivalent directions. In the step-flow growth mode, the faster growth speed in the direction normal to a type $A$ edge will result in a jagged shaped edge. Each protrusion of the jagged edge is bounded by type $B$ edges. On the other hand, the slower growth speed type $B$ edge will

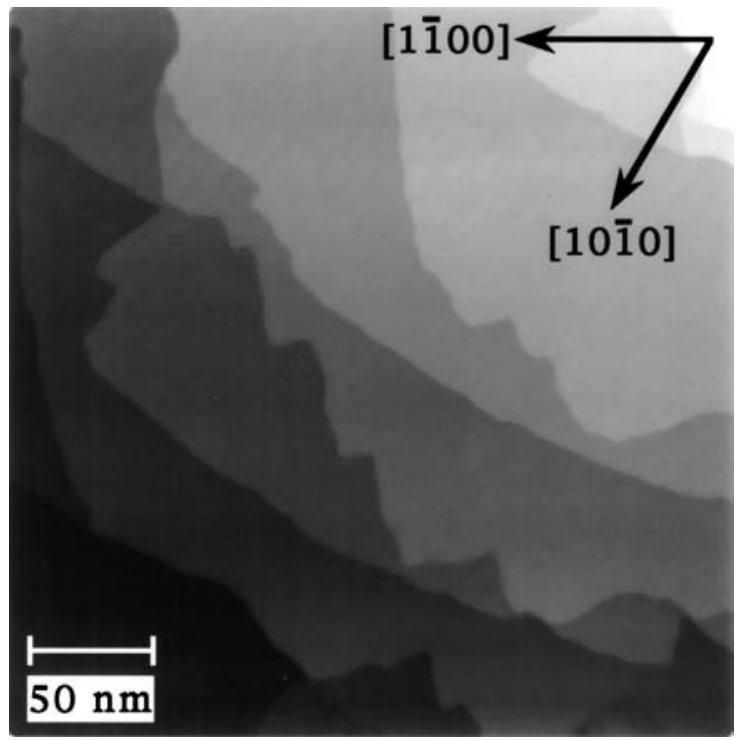

FIG. 1. STM picture of step-flow growth mode of $\mathrm{GaN}$ at substrate temperature $=650{ }^{\circ} \mathrm{C}$, sample bias $-2.5 \mathrm{~V}$, and tunneling current $0.1 \mathrm{nA}$. be smooth. To explain the alternating smooth-jagged sequence of the terrace edges, we refer to Fig. 2(b), where we show that a hcp film has alternating type $A$ and $B$ edges along a given crystallographic symmetry direction. However, at $\pm 60^{\circ}$ or $180^{\circ}$ away, the order of the type $A$ and $B$ edges is reversed, exactly corresponding to the observations of the STM picture. A corollary to this description is that a smooth-jagged edge ordering is evidence that the terraces are separated by a single (or odd multiples) bilayer height. STM measurements of the terrace heights in Fig. 1 confirm that all the terraces are separated by a single bilayer height.

The growth anisotropy will lead to step bunching in a hcp film, because the fast-growing steps will ultimately catch up with the slow ones as growth proceeds. This process will lead to qualitatively different edge appearances between a cubic and hexagonal film. Referring to Fig. 2(b), for a fcc(111) film, the type $A$ edge points always along the $\left[\begin{array}{ll}1 & 1\end{array}\right]$ and equivalent directions on all terraces while the type $B$ edge points always along the [2 111$]$ and equivalent directions. Thus, in a step-flow growth mode for a fcc(111) film, step bunching does not occur, and the appearance of smooth and jagged edges will remain on each terrace [see Fig. 3(a)]. On the other hand, for a hcp(0001) film, all terrace edges will eventually become smooth when the fast-growing type $A$ edges catch up with the slow-growing type $B$ edges. Thus, if one descends the terraces along the $\left[\begin{array}{lll}1 & 0 & 1\end{array}\right]$ direction,

(a)

(b)
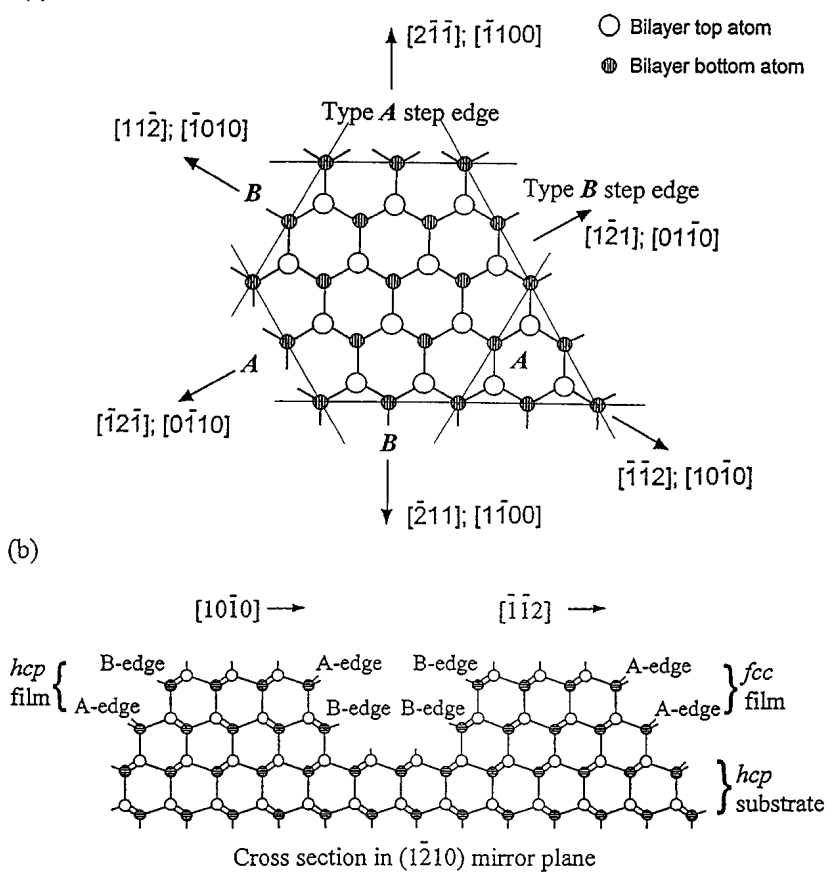

FIG. 2. (a) Plan view of a bilayer, showing type $A, B$ edges and fcc, hcp directions. For a hcp direction, the edge may be either $A$ or $B$, depending on the stacking sequence. (b) Crosssectional view of hcp, fcc films on a hcp substrate. 
(a) $f c c(111)$

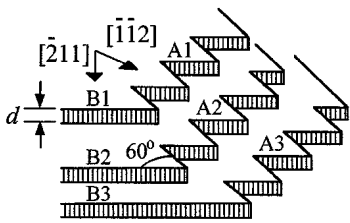

(b) $h c p(0001)$

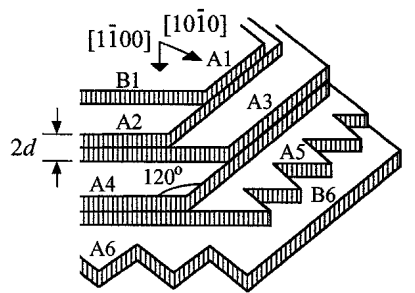

FIG. 3. Terrace step shapes due to step-flow growth anisotropy for (a) a fcc film and (b) a hcp film.

one will encounter double height steps. On the other hand, if one descends the terraces along the $\left[\begin{array}{llll}1 & \overline{1} & 0 & 0\end{array}\right] \mathrm{di}-$ rection and after descending each terrace, alternately turning $\pm 60^{\circ}$, one will encounter only single bilayer step heights [see Fig. 3(b)]. The strikingly different appearance in the terrace edge shapes between a fcc(111) film and a hcp(0001) film is a direct consequence of growth anisotropy. The step bunching and smooth terrace edges are indeed observed experimentally, as is shown in Fig. 4. Double bilayer steps have emerged at the expense of single bilayer ones. Some step edges are extremely smooth, with dimensions as long as $600 \AA$.

Lowering the temperature leads to a growth mode change from step-flow to 2D nucleation. This is due to the reduction of diffusion length of adatoms on the surface. As explained before, the anisotropic growth speeds of type $A$ and $B$ edges will result in triangularly shaped islands. Figure 5 shows a STM picture of $\mathrm{GaN}(0001)$ grown at $500{ }^{\circ} \mathrm{C}$ on top of the $650{ }^{\circ} \mathrm{C}$ buffer. The growth is monitored by RHEED intensity oscillations [8], and is stopped at the trough of the 5th oscillation. The observation of RHEED intensity oscillations indicates

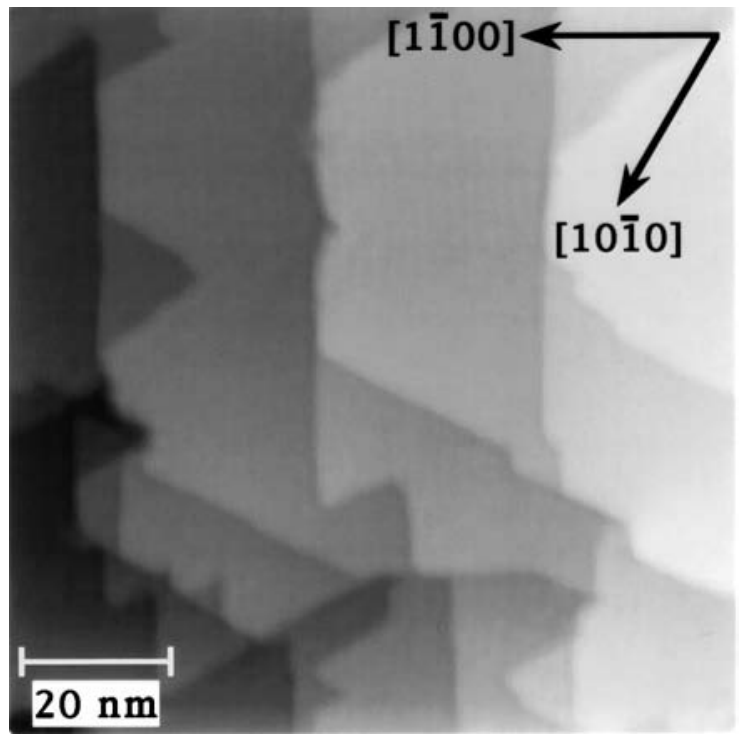

FIG. 4. STM picture of GaN step-flow growth mode at $650{ }^{\circ} \mathrm{C}$ showing step bunching. Other conditions are the same as Fig. 1. island nucleation growth, which is confirmed by the STM image of Fig. 5 showing islands on terraces. As expected, the islands having the triangular shape are observed. Measurement of height differences by STM shows that the terraces have single bilayer height differences and the triangular islands have single bilayer heights on the terraces. However, we also observe triangular islands of the same height on the same terrace which are oriented $180^{\circ}$ from each other. For single bilayer height hcp islands on terraces separated by a bilayer step height, their orientations should be $180^{\circ}$ rotated, as is clear from the atomic model shown in Fig. 2(b). In order for $180^{\circ}$ rotated triangular islands to appear on the same terrace, they must be either: (i) hcp islands with a bilayer height difference, or (ii) the coexistence of hcp and fcc islands of the same height. From the STM picture, we can rule out case (i) and conclude that the island growth mode has resulted in a mixture of hcp and fcc oriented islands. We have checked this with cross-sectional high resolution transmission electron microscopy (HRTEM) and the TEM image confirms the presence of cubic phase regions embedded in the bulk hexagonal phase.

We now comment on the assumption that the growth speed of a type $A$ edge is greater. For a hcp(0001) film, because of the alternating stacking sequence, the observation of jagged step edges or triangular island vertices pointing in the $\left[\begin{array}{llll}1 & 0 & 1 & 0\end{array}\right]$ direction does not prove that a type $A$ edge grows faster. However, if triangular islands are observed on a fcc(111) film and if the vertex direction is identified, then the observation will prove which step edge grows faster. Avery et al. [11] have recently shown STM pictures which include that of island growth on a GaAs(111) film. Although these authors did not comment on the island shape, it is clear from their data that apart from some roughness, most islands are indeed triangularly shaped with vertices pointing in the $\left[\begin{array}{ll}1 & 1\end{array}\right]$ and

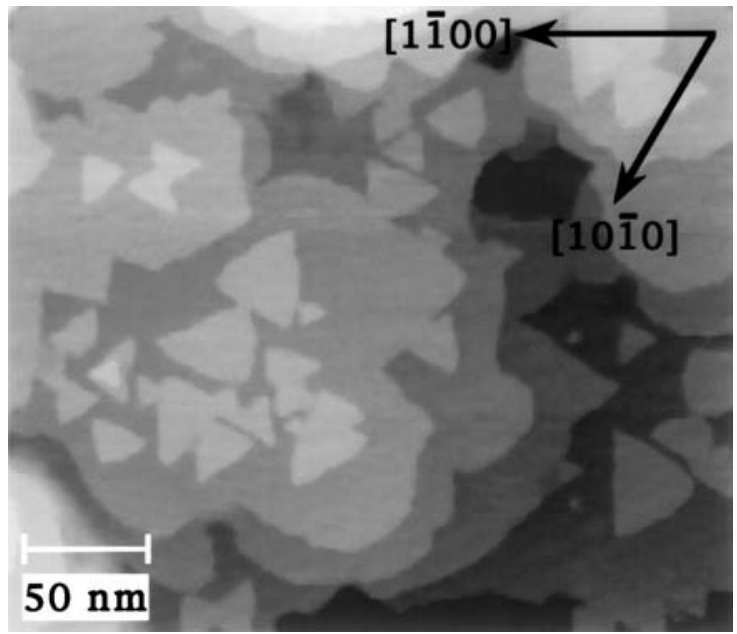

FIG. 5. STM picture of island nucleation of $\mathrm{GaN}$ at $500^{\circ} \mathrm{C}$. Other conditions are the same as Fig. 1. 
equivalent directions. Their data confirm that the assumption made here that type $A$ edges grow faster is correct. Furthermore, as expected from our model for a cubic film, the islands imaged by Avery et al. [11] on different terraces of the GaAs(111) film all have the same orientation. To address the issue of whether the edge-growth anisotropy is due to adatom/edge-atom bonding or subsurface layer influence on the adatom mobility, we observe that although a hcp island and fcc island on a given terrace have different subsurface stacking geometries, the islands nevertheless have similar sizes and qualitatively similar triangular shapes (but $180^{\circ}$ misoriented). This observation suggests that the growth anisotropy in hcp and fcc $\mathrm{GaN}$ films is dominated by anisotropic edge accommodation, similar to that found by Mo et al. [6] and others [12] for homoepitaxy on $\mathrm{Si}(001)$. The same mechanism has been attributed to causing step bunching in polytype $\mathrm{SiC}(0001)$ growth [13]. It seems we now have evidence that growth anisotropy in semiconductors is mainly due to anisotropic adatom accommodation at an edge, a process different from what is found in metal homoepitaxy, where the dominant effect is due to the anisotropic subsurface induced diffusion rate [7,14].

Instead of a conclusion, we offer a discussion of the implications of the above results. In the $2 \mathrm{D}$ island growth mode, we have observed the coexistence of both hcp and fcc islands on the same terrace. Eventually, the islands will coalesce. Coalesced islands can easily be seen in Fig. 5. When this happens, the coalesced islands will inevitably create line or plane defects due to their boundaries' mismatch. The resulting film will be inferior in structural order. If, on the other hand, one grows in the step-flow mode, formation of the mixed phases can be suppressed by the atomic structure at the step. The STM pictures in Figs. 1 and 4 show that the entire $1000 \times 1000 \AA^{2}$ area is of the hcp phase. Adatoms, once incorporated, will follow this structure, which will lead to a pure phase and a more uniform film. Lattice mismatch between substrate and film creates misfit dislocations with edge components which run parallel to the interface. These dislocations are usually connected to threading arms with vertical components. Most threading arms cross the entire thickness of the film and are harmful to the quality of the film. If a threading dislocation is of the screw type with Burgers vector $\mathbf{b}=\mathbf{c}=5.18 \AA$, it can be an effective nucleation center which causes double spiral growth. The edges of the spirals form single bilayer steps as shown in Fig. 1. Thus, a strategy to reduce the number of screw nucleation centers is to maintain the step flow growth from a vicinal substrate. With a large enough diffusion length, atom incorporation at step edges dominates and nucleation centers on the terraces themselves are greatly suppressed. The step flow growth may also lengthen parallel-running edge dislocation lines, resulting in a reduction in the number of threading dislocations with vertical components. Thus, the quality of the film far from the interface is improved [15]. Another longdistance vertical defect is inversion domain boundaries (IDBs) [16]. To minimize IDBs, the starting substrate should have atomically smooth terraces with the same elemental termination. Because $4 H$ or $6 H \mathrm{SiC}(0001)$ is nonisomorphic with $2 H-\mathrm{GaN}(0001)$, stacking mismatch boundaries (SMBs) or double positioning boundaries (DPBs) [17,18] will occur at step edges between the substrate and the film. However, SMBs (or DPBs) are short-distance vertical defects because they can be cured by stacking faults in the nitride film; thus, away from the interface, the film quality is good. Details of morphological studies of GaN films grown in the step flow mode on vicinal $\mathrm{SiC}(0001)$ substrates with atomically smooth terraces will be reported elsewhere [15].

This work is supported in part by HK RGC Grants No. HKU 7118/98P, No. 7117/98P, No. HKU 260/95P, U.S. DOE Grant No. DE-FG02-84ER 45076, and NSF Grant No. DMR-9214054.

[1] S. Strite and H. Morkoc, J. Vac. Sci. Technol. B 10, 1237 (1992).

[2] S. Nakamura, M. Senoh, S.-I. Nagahama, N. Iwasa, T. Yamada, T. Matsushita, Y. Sugimoto, and H. Kiyoku, Appl. Phys. Lett. 70, 1471 (1997).

[3] S. Nakamura, Jpn. J. Appl. Phys. 30, L1705 (1991).

[4] A. R. Smith, R. M. Feenstra, D. W. Greve, J. Neugebauer, and J. E. Northrup, Phys. Rev. Lett. 79, 3934 (1997).

[5] A. Pavlovska, E. Bauer, V.M. Torres, J. L. Edwards, R. B. Doak, I. S. T. Tsong, V. Ramachandran, and R. M. Feenstra, J. Cryst. Growth 189/190, 310 (1998).

[6] Y.-W. Mo, B. S. Swartzentruber, R. Kariotis, M. B. Webb, and M. G. Lagally, Phys. Rev. Lett. 63, 239 (1989).

[7] T. Michely, M. Hogage, M. Bott, and G. Comsa, Phys. Rev. Lett. 70, 3943 (1993).

[8] M.H. Xie, S.M. Seutter, W. K. Zhu, L.X. Zheng, Huasheng $\mathrm{Wu}$, and S. Y. Tong (to be published).

[9] Y. Nakada and H. Okumura, J. Cryst. Growth 189/190, 370 (1998).

[10] A. R. Smith, R. M. Feenstra, D. W. Greve, M. S. Shin, M. Skowronski, J. Neugebaner, and J.E. Northrup, Appl. Phys. Lett. 72, 2114 (1998).

[11] A. R. Avery, H. T. Dobbs, D. M. Holmes, B. A. Joyce, and D. D. Vvedensky, Phys. Rev. Lett. 79, 3938 (1997).

[12] J. Y. Tsao, E. Chason, U. Koehler, and R. Hamers, Phys. Rev. B 40, 11951 (1989).

[13] T. Kimoto, A. Itoh, H. Matsunami, and T. Okano, J. Appl. Phys. 81, 3494 (1997).

[14] R. T. Tung and W. R. Graham, Surf. Sci. 97, 73 (1980).

[15] M.H. Xie, L.X. Zheng, C.H. Pang, Huasheng Wu, N. Ohtani, and S. Y. Tong (to be published).

[16] J. E. Northrup, J. Neugebauer, and L. T. Romano, Phys. Rev. Lett. 77, 103 (1996).

[17] B. N. Sverdlov, G. A. Martin, H. Morkoc, and D. J. Smith, Appl. Phys. Lett. 67, 2063 (1995).

[18] S. Tanaka, R. S. Kern, and R. F. Davis, Appl. Phys. Lett. 66, 37 (1995). 